Journal of Engineering and Applied Sciences 7 (3): 290-294, 2012

ISSN: 1816-949X

(C) Medwell Journals, 2012

\title{
Analyzing of Optical Band Gap in Bis Glycine Cobalt Sulfate Single Crystal
}

\author{
${ }^{1} \mathrm{G}$. Anbazhagan, ${ }^{2} \mathrm{P}$.S. Joseph, ${ }^{3} \mathrm{G}$. Shankar and ${ }^{4}$ T. Balakrishnan \\ ${ }^{1} \mathrm{PG}$ and Research Department of Physics, H.H. The Rajah's College, 622001 Pudukottai, India \\ ${ }^{2} \mathrm{PG}$ and Research Department of Physics, Thanthai Hans Roever College, 621212 Perambular, India \\ ${ }^{3}$ Department of Physics, Government College of Engineering, 636011 Salem, India \\ ${ }^{4} \mathrm{PG}$ and Research Department of Physics, Periyar EVR College, 620023 Tiruchirappalli, India
}

\begin{abstract}
Single crystals are Bis Glycine Cobalt Sulfate (BGCS) recently investigated nonlinear optical materials were grown by slow evaporation techniques. Optical absorption of the crystals of BGCS has been measured at room temperature from which the optical band gap has been determined using the absorption spectrum as $5.90 \mathrm{eV}$. Vibrational modes of grown crystals were conformed by FTIR spectrum. The optical absorption spectrum shows that the UV cut-off wavelength for the grown crystal is at $240 \mathrm{~nm}$. The optical constant $(\alpha, \mathrm{K}$, R) were also evaluated.
\end{abstract}

Key words: BGCS, optical band gap, optical constants, FTIR spectrum, crystal, India

\section{INTRODUCTION}

Due to unique properties the Nonlinear Optical (NLO) single crystals have promising applications in the area of photonics such as high speed information process, frequency conservation, optical communication, high optical disc data storage, etc. (Shankar et al., 2010; Prasad, 1991; Marder et al., 1991; Saleh and Teich, 1991; Penn et al., 1991). Organic molecules especially chalcones are of particular interest over inorganic molecules in the field Nonlinear Optical (NLO) applications (Dongol, 2002; Chemla and Zyss, 1987; Zyss, 1993; Karna, 2000). The crystals were excellent physical properties and good record for long term stability in devices.

Considerable interest has been evinced by researchers in crystals semiconductor for use in solar cell devices and thin crystal transistor for the plat panel displays (Chandra et al., 1980; Chopra and Das, 1983; Miniewicz and Bartkiewicz, 1993; Kajzar et al., 1995; Shankar and Varma, 1996; Ester and Halfpenny, 1998; Kejalakshmy and Srinivasan, 2004). Tuning of optical band gap in semiconductor materials is an important tool in optoelectronics and photonics integration. The optical behaviors of materials are essential parameters to determine its usage in optoelectronic devices (Pandey et al., 2005). Knowledge of optical constants of a material such as optical band gap and extension coefficient is vital to scrutinize the materials potential for optoelectronic applications (Dongol, 2002). The electrical properties of semiconductor are strongly dependent up on the band gap (Beyer et al., 1971).
The crystal growth is a frontier area of science and technology which plays a major role in the technology of photonics (Vesta et al., 2009). The past development in the field of optoelectronic and photonic and necessity. The search for new and efficient Nonlinear Optical (NLO) materials. That can be utilized for optical computing, optical communication, electro optics, frequency shifting, optical data storage for developing technologies in telecommunications (Kumar et al., 2009). The report here the growth and characterization of Bis Glycine Cobalt Sulfate (BGCS) crystal, Fourier Transform Infrared (FTIR) Spectroscopy, optical transmission and optical absorption studies, the optical constant $(\alpha, \mathrm{K}, \mathrm{R})$ are calculated (Gaffar et al., 2003). Finally, the optical band gap was estimated form the optical absorption spectrum (Mardare and Rusu, 2001).

\section{MATERIALS AND METHODS}

Crystal synthesis: The saturated solution (BGCS) crystal was prepared at room temperature and stirred well to enable homogenizes of the solution the crystals were prepared by slow cooling method. BGCS single crystal were grown by dissolving high purity of glycine cobalt sulfate in the ratio $2: 1$ and stirred well for about $3 \mathrm{~h}$. After a period of 10-12 days, single crystals of BGCS were grown by slow evaporation techniques having dimension $9 \times 5 \times 3 \mathrm{~mm}^{3}$ at room temperature. Figure 1 shows the photograph of grown crystal of Bis Glycine Cobalt Sulfate (BGCS). The crystals were subject to spectral analysis for the investigation of structural and optical properties. The

Corresponding Author: G. Shankar, Department of Physics, Government College of Engineering, 636011 Salem, India 


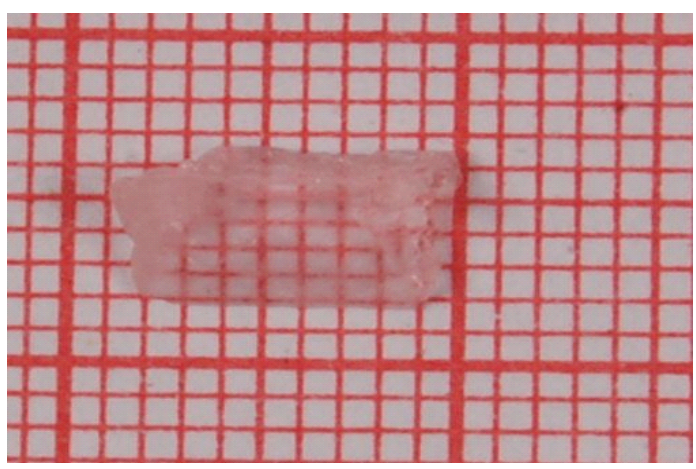

Fig. 1: Photo of BGCS crystal

optical properties of the crystal were studied by the UV-spectrum, using Lambda 35 Spectrophotometer. The optical constants were calculated using the theoretical formulae. The functional group was analyzed using FTIR Spectrum. The spectral variation of the optical absorption coefficient $(\alpha)$, extinction coefficient $(\mathrm{K})$, optical reflectance $(R)$ and optical band gap $\left(E_{g}\right)$ also calculated from the obtained optical transmittance and reflectance value.

\section{RESULTS AND DISCUSSION}

FTIR analysis: The FTIR spectrum was measured within the $400-4000 \mathrm{~cm}^{-1}$ region using Perkin Elmer FTIR Spectrophotometer by KBr Pellet Technique. The FTIR spectrum of PMP crystal is shown in Fig. 2. The peak at $3332 \mathrm{~cm}^{-1}$ is due to $\mathrm{OH}$ stretch of water. The peak at $3263 \mathrm{~cm}^{-1}$ is due to $\mathrm{NH}_{3}$ asymmetric stretching. $\mathrm{C}=\mathrm{O}$ asymmetric stretching is observed at $1603 \mathrm{~cm}^{-1}$. C $=\mathrm{O}$ symmetric stretching is observed at $1389 \mathrm{~cm}^{-1}$. The peaks at 1118,1176 and $672 \mathrm{~cm}^{-1}$ indicates the presences of S-O stretching. The peak observed at $1322 \mathrm{~cm}^{-1}$ is due to the $\mathrm{CH}_{2}$ wagging vibrations. The $\mathrm{CH}$ out of plane deformation is observed at $919 \mathrm{~cm}^{-1}$. The peak at $743 \mathrm{~cm}^{-1}$ is due to $\mathrm{OH}$ deformation.

UV-Vis spectroscopy analysis: To calculate the optical band gap, the absorption spectra of grown crystals were recorded. First the optical absorption coefficient $(\alpha)$ was calculated form the absorption using the relation: $\alpha=$ absorption/t where, $t$ is the thickness of the crystal, the relation between the optical band gap $\left(\mathrm{E}_{\mathrm{g}}\right)$ and the optical absorption coefficient $(\alpha)$, near the absorption edge can be written as (Ashour et al., 1995):

$$
\alpha=\frac{\mathrm{A} \sqrt{\left(\mathrm{h} v-\mathrm{E}_{\mathrm{g}}\right)}}{\mathrm{h} v}
$$

Where:

$\mathrm{A}=$ The constant

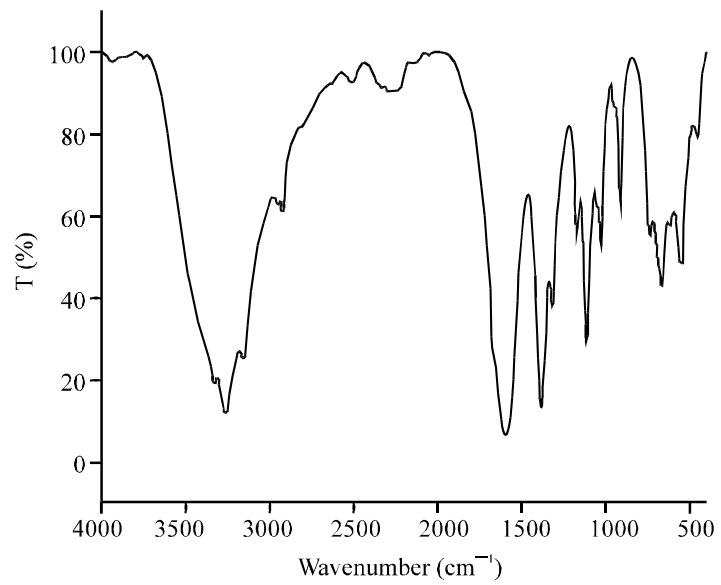

Fig. 2: FTIR analysis of BGCS crystal

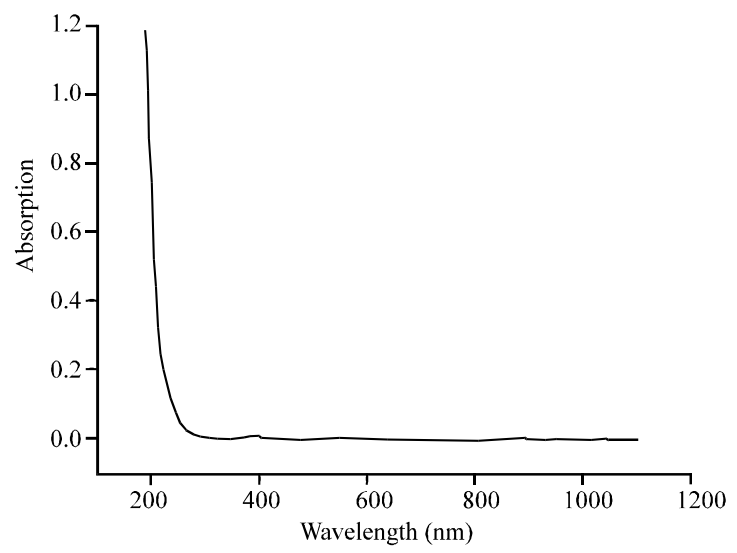

Fig. 3: Optical absorption spectrum of BGCS crystal

$\mathrm{E}_{\mathrm{g}}=$ The optical band gap

$\mathrm{h}=$ The Plank's constant

$v=$ The frequency of incident photon

The plot of $(\alpha h v)^{2}$ verses photon energy is shown in Fig. 3. The band gap of the grown crystal was obtained as $5.90 \mathrm{eV}$ from the intercept on the $\mathrm{x}$-axis with the extrapolated straight line. The transparency of the grown crystal was found to be good agreement with reported one (Kumar et al., 2011). This is the most favorable characteristic for NLO materials.

The optical transmission of BGCS crystal was measured using Lambda 35 Spectrophotometer. It was assured that the BGCS crystal as good transmittance near $75 \%$ and the lower cut off wavelength of the crystal is found to be $240 \mathrm{~nm}$. High transmittance with lower cut off wavelength was comparatively good with reference reported (Peter and Ramasamy, 2010; Meijerink et al., 1990).

A good optical transmittance is very desirable in an NLO crystal. Since, the absorption if any NLO materials 


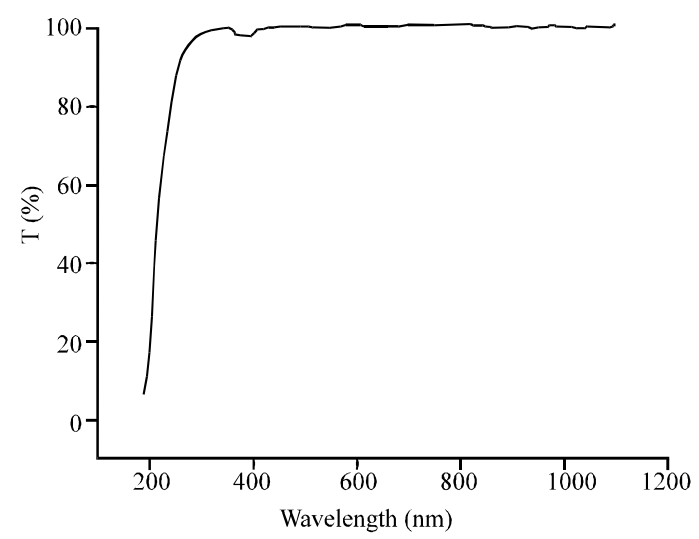

Fig. 4: Optical transmission spectrum of BGCS crystal

near the fundamental of the second harmonic will lead to less conversion efficiency in those wavelengths. When absorption is maintain from shorter wavelength to longer wavelength, the enhanced transmission is absorption between 240 and $1100 \mathrm{~nm}$ as the entire region does not bear any absorption band it can be used NLO applications. A plot of variation of absorption verses wavelength is shown in Fig. 3. From the transmission spectrum, it is evident that BGCS as a UV lower cut off wavelength at $240 \mathrm{~nm}$ and the transparency in the entire visible region for this crystals. A plot of variation of transmittance verses wavelength is shown in Fig. 4. Suggest its suitability for second harmonic generation. (Eazhilarasi et al., 2008).

The depends of optical absorption coefficient with the photon energy helps to study the band structure and the type of transition of electrons (Lenin et al., 2009). The optical absorption coefficient $(\alpha)$ was calculated from the transmittance using the following relation:

$$
\alpha=\frac{2.3036 \log (1 / \mathrm{T})}{\mathrm{d}}
$$

Where:

$\mathrm{T}=$ The transmittance

$\mathrm{d}=$ The thickness of the crystal

In the high energy region, the energy dependents of absorption coefficient such as the occurrence of direct band gap. A direct band gap semiconductor, the crystal under study of as an absorption coefficient obeying the relation:

$$
\alpha=\frac{\mathrm{A} \sqrt{\left(\mathrm{h} v-\mathrm{E}_{\mathrm{g}}\right)}}{\mathrm{h} v}
$$

Where:

$\mathrm{E}_{\mathrm{g}}=$ The optical band gap of the crystal

$\mathrm{A}=$ The constant

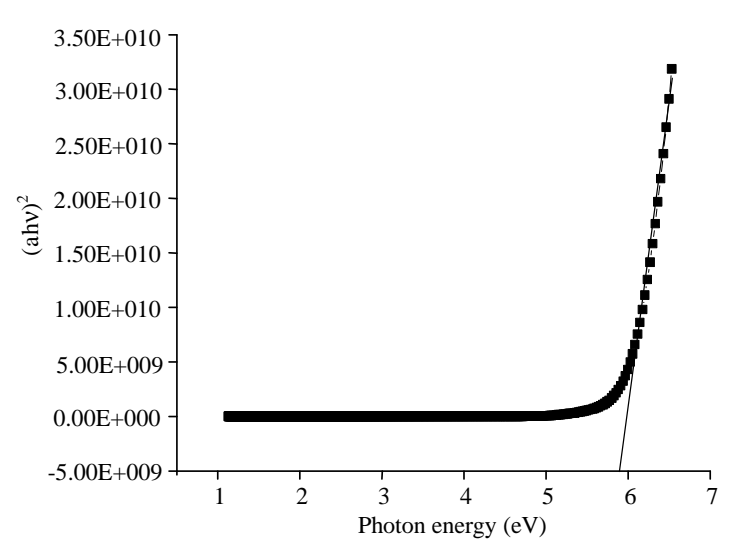

Fig. 5: Plot of $(\alpha h v)^{2}$ verses photon energy for spectrum of BGCS crystal

A plot of variation $(\alpha h v)^{2}$ verses photon energy is shown in Fig. 5. $\mathrm{E}_{\mathrm{g}}$ was evaluated by the interpolation of the linear part. The band gap is found to $5.90 \mathrm{eV}$ as a consequent of wide band gap, the crystal under study as a large transmittance in the visible region (Periyasamy et al., 2007).

Optical constants measurement: The refractive index can be determined (Gupta and Mansingh, 1996) from the Reflectance (R) data using:

$$
\mathrm{R}=\frac{(\mathrm{n}-1)^{2}}{(\mathrm{n}+1)^{2}}
$$

The Transmittance $(\mathrm{T})$ is given by:

$$
\mathrm{T}=\frac{(1-\mathrm{R})^{2} \exp (-\alpha \mathrm{d})}{1-\mathrm{R}^{2} \exp (-2 \alpha \mathrm{d})}
$$

The optical extinction coefficient can be obtained from the following relation:

$$
\mathrm{K}=\frac{\alpha \lambda}{4 \pi}
$$

Where:

$\lambda=$ Wavelength of source

$\alpha=$ The optical absorption coefficient of the crystal

The reflectance in terms of the absorption coefficient can be derived from the above equations. Hence:

$$
\mathrm{R}=\frac{1 \pm \sqrt{1-\exp (-\alpha \mathrm{d})+\exp (\alpha \mathrm{d})}}{1+\exp (-\alpha \mathrm{d})}
$$

Where:

$\mathrm{d}=$ The thickness of the crystal 


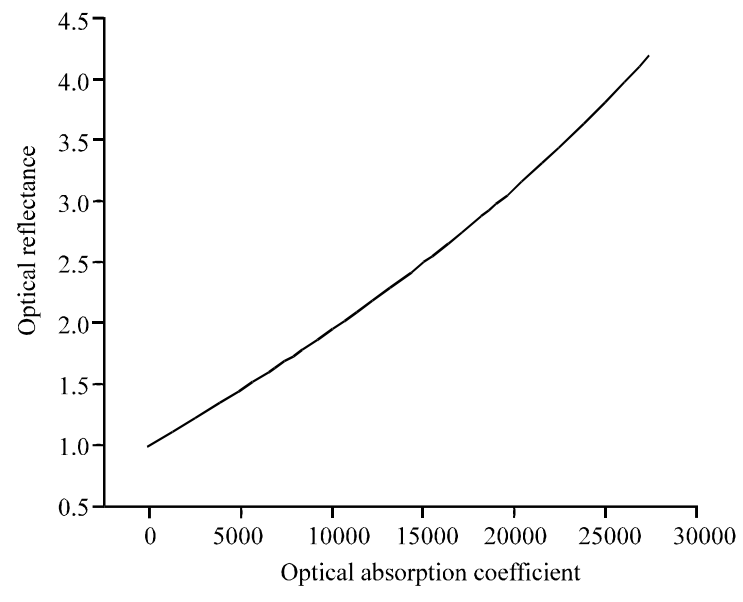

Fig. 6: Plot of optical absorption coefficient verses optical reflectance of BGCS cystal

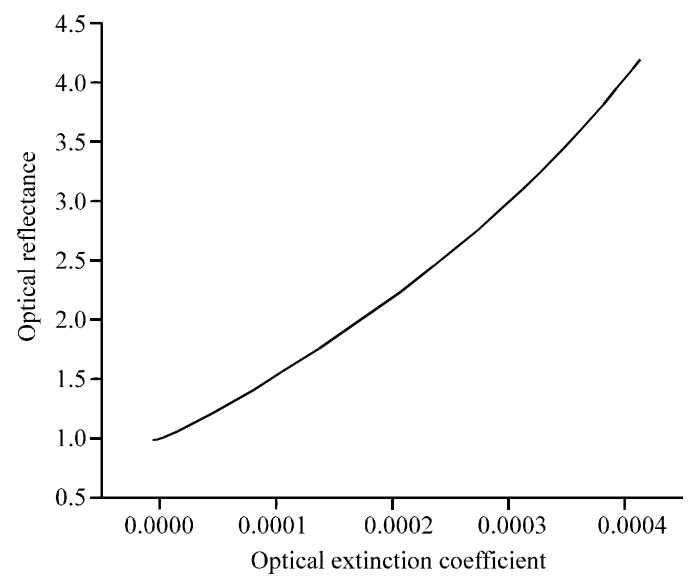

Fig. 7: Plot of optical extinction coefficient verses optical reflectance of BGCS crystal

$\mathrm{n}=$ The refractive index

$\alpha=$ The optical absorption coefficient of the crystal

The optical absorption coefficient verses optical reflectance graph is shown in Fig. 6. From the above data, the refractive index $\mathrm{n}$ can also be derived as:

$$
\mathrm{n}=\frac{-(\mathrm{R}+1) \pm \sqrt{-3 \mathrm{R}^{2}+10 \mathrm{R}-3}}{2(\mathrm{R}-1)}
$$

Figure 7 shows the variation of reflectance $(\mathrm{R})$ and extinction coefficient $(\mathrm{K})$ as a function of absorption coefficient, respectively. From Fig. 7, it is clear that both the reflectance and extinction coefficient depend on the absorption coefficient. The internal efficiency of tailoring the absorption coefficient and tuning the band gap of the material, researchers can achieve the desired material which is suitable for fabricating various layers of the optoelectronic and photonic devices as per requirements (Krishnakumar and Nagalakshmi, 2005; Krishnakumar and Xavier, 2004; Krishnan et al., 2008).

\section{CONCLUSION}

Good quality BGCS crystal was synthesis and subjected to FTIR and optical studies. FTIR analysis was carried out to conform the presence of functional groups of the grown crystal. The optical transmittance of the crystal conform the transparency of the crystal. By tailoring the absorption coefficient and tuning the band gap of the materials, researchers can active the desired material which is suitable for the fabrication of various optoelectronic devices because of its wide optical band gap. The value of the optical constant, absorption coefficient, extinction coefficient and optical reflectance such as that the BGCS crystals are suitable for the fabrication of various optoelectronic and photonic devices.

\section{REFERENCES}

Ashour, A., N. El-Kadry and S.A. Mahmoud, 1995. On the electrical and by a modified source. Thin Solid Films, 269: $117-120$.

Beyer, W., H. Mell and J. Stuke, 1971. Conductivity and thermoelectric power of trigonal $\mathrm{Se}_{\mathrm{z}} \mathrm{Te}_{1 \cdot \mathrm{x}}$ single crystals. Phys. Status Solidi B, 45: 153-162.

Chandra, S., R.K. Pandey and R.C. Agrawal, 1980. Solar energy conversion by photoelectrochemical cells using chemical-bath-deposited CdS films. J. Phys. D: Applied Phys., Vol. 13. 10.1088/0022-3727/13/9/025

Chemla, D.S. and J. Zyss, 1987. Nonlinear Optical Properties of Organic Molecules and Crystals. Vol. 12, Academic Press, New York.

Chopra, K.L. and S.R. Das, 1983. Thin Film Solar Cells. Plenum press, New York, USA.

Dongol, M., 2002. Optical absorption and structural properties of as-deposited and thermally annealed As-Te-Ga thin films. Egypt. J. Solids, 25: 33-47.

Eazhilarasi, G., R. Nagalakshmi and V. Krishnakumar, 2008. Studies on crystal growth, vibrational and optical properties of organic nonlinear optical crystal: $\mathrm{p}$ Aminoazobenzene. Spectrochimica Acta Part A Mol. Biomol. Spectroscopy, 71: 502-507.

Ester, G.R. and P.J. Halfpenny, 1998. Observation of twodimensional nucleation on the $\left\{\begin{array}{lll}0 & 1 & 0\end{array}\right\}$ face of potassium hydrogen phthalate (KAP) crystals using ex situ atomic force microscopy. J. Cryst. Growth, 187: 111-118. 
Gaffar, M.A., A. A. El-Fadl and S.B. Anooz, 2003. Influence of strontium doping on the indirect band gap and optical constants of ammonium zinc chloride crystals. Phys. B Condens. Matter, 327: 43-54.

Gupta, V. and A. Mansingh, 1996. Influence of postdeposition annealing on the structural and optical properties of sputtered zinc oxide film. J. Applied Phys., 80: 1063-1073.

Kajzar, F., A. Lorin, J. Le Moigne and J. Szpunar, 1995. Habit modification of KAP single crystals by impurities. Acta Phys. Pol. A, 87: 713-713.

Karna, S.P., 2000. Electronic and nonlinear optical materials: The role of theory and modeling. J. Phys. Chem. A, 104: 4671-4673.

Kejalakshmy, N. and K. Srinivasan, 2004. Growth, optical and electro-optical characterisations of potassium hydrogen phthalate crystals doped with $\mathrm{Fe}^{3+}$ and $\mathrm{Cr}^{3+}$ ions. Opt. Mater., 27: 389-394.

Krishnakumar, V. and R. Nagalakshmi, 2005. Crystal growth and vibrational spectroscopic studies of the semi organic non-linear optical crystal-bisthiourea zinc chloride. Spectrochimica Acta Part A: Mol. Biomol. Spectroscopy, 61: 499-507.

Krishnakumar, V. and R.J. Xavier, 2004. FTRaman and FT-IR spectralstudies of 3-mercapto-1,2,4-triazole. Acta Part A: Mol. Biomol. Spectroscopy, 60: 709-714.

Krishnan, S., C.J. Raj, S. Dinakaran and S.J. Das, 2008. Investigation of optical band gap in potassium acid phthalate single crystal. Cryst. Res. Technol., 43: 670-673.

Kumar, S.M.R., N. Melikechi, S. Selvakumar and P. Sagayaraj, 2009. Growth and characterization of non-linear optical crystal. Cryst. Res. Technol., 43: $670-673$.

Kumar, R.A., N. Sivakumar, R.E. Vizhi and D.R. Babu, 2011. The effect of $\mathrm{Fe}^{3+}$ doping in potassium hydrogen phthalate single crystals on structural and optical properties. Phys. B: Conden. Matter, 406: 985-991.

Lenin, M., G. Bhagavannarayana and P. Ramasamy, 2009. Synthesis, growth and characterization of a nonlinear optical crystal-glycine lithium chloride. Opt. Commun., 282: 1202-1206.

Mardare, D. and G.I. Rusu, 2001. On the structure and optical dielectric constants of $\mathrm{TiO}_{2}$ sputtered thin films. J. Opto. Electron. Adv. Mater., 3: 95-100.
Marder, S.R., J.E. Sohn and G.D. Stucky, 1991. Materials for Nonlinear Optics: Chemical Perspectives. American Chemical Society, Washington, DC., USA., ISBN-13: 9780841219397 , Pages: 766.

Meijerink, A., G. Blasse and M. Glasbeek, 1990. Photoluminescence, thermoluminescence and EPR studies on Zn4B6013. J. Phys. Condense. Matter, 2: 6303-6323.

Miniewicz, A. and S. Bartkiewicz, 1993. On the electrooptic properties of single crystals of sodium, potassium and rubidium acid phthalates. Adv. Mater. Opt. Electron., 2: 157-163.

Pandey, V., N. Mehta, S.K. Tripathi and A. Kumar, 2005. Optical band gap and optical constants in $\mathrm{Se}_{85} \mathrm{Te}_{15}$ $\mathrm{xPb}_{\mathrm{x}}$ thin films. J. Optoelectron. Adv. Mater., 7: 2641-2646.

Penn, B.G., B.H. Cardelino, C.E. Moore, A.W. Shields and D.O. Frazier, 1991. Growth of bulk single crystals of organic materials for nonlinear optical devices: An overview. Prog. Cryst. Growth Charact. Mater., 22: 19-510.

Periyasamy, B.K., R.S. Jebas, N. Gopalakrishanan and T. Balasubramanian, 2007. Development of NLO tunable band gap organic devices for optoelectronic applications. Mater. Lett., 61: 4246-4249.

Peter, M.E. and P. Ramasamy, 2010. Synthesis, growth and characterization of a novel semiorganic NLO crystal: Triglycine calcium dibromide. Spectrochim, Acta A., 75: 1417-1421.

Prasad, P.N., 1991. Polymeric materials for non-linear optics and photonics. Polymer, 32: 1746-1751.

Saleh, B.E.A. and M.C. Teich, 1991. Fundamentals of Photonics. John Wiley and Sons Inc., New York, USA., ISBN-13: 9780471839651 , Pages: 966.

Shankar, G., P.S. Joseph, M. Yosuva Suvakin and A. Sebastiyan, 2010. Investigation of optical band gap in pyrrolidinomethylphthalimide crystal. Phys. B: Phys. Condens. Matter, 405: 4231-4234.

Shankar, M.V. and K.B.R. Varma, 1996. Piezoelectric resonance in KAP single crystals. Ferroelectr. Lett., 21: $55-59$.

Vesta, C., R. Uthrakumar, B. Varghese, S.M.N. Priya and S.J. Das, 2009. Growth, structural investigation and characterization on novel organic NLO single crystal: Tri-nitrophenol para hydroxyacetophenone. J. Cryst. Growth, 311: 1516-1520.

Zyss, J., 1993. Molecular Nonliner Optics. Academic Press, Boston. 